\title{
On the QoS-capable transport in the IP-based UTRAN
}

\author{
A. Samhat, T. Chahed \\ GET/Institut National des Télécommunications \\ Département Réseaux et Services des Télécommunications \\ 9 rue Charles Fourier - 91011 Evry CEDEX - France \\ e-mail : \{abedellatif.samhat, tijani.chahed\}@int-evry.fr
}

\begin{abstract}
In this paper, we highlight different architectures and techniques for transport of user traffic in the IP-based UMTS Terrestrial Radio Access Network (UTRAN) taking into account the real-time nature of the transport therein. Indeed, the UTRAN imposes severe delay bounds on the transport of both real-time and non real-time traffic. In this context, we provide an overview of different QoS-capable transport architecture options, such as DiffServ and MPLS. We also discuss multiplexing schemes that shall reduce overheads and hence achieve an efficient utilization of limited wired resources. We investigate these transport options and their relative merits taking into account several factors including transport infrastructure and network topology.
\end{abstract}

Index Terms-UTRAN, IP-based transport, QoS, DiffServ, MPLS, Multiplexing.

\section{INTRODUCTION}

Universal Mobile Telecommunications System (UMTS) is one of the $3 \mathrm{G}$ cellular systems standardized by the Third Generation Partnership Project (3GPP). It is a multi-service system enabling multimedia services to users anytime, anywhere. An intensive research activity is currently investigating the basic design of UMTS, and a significant amount of this activity focuses on the UMTS Terrestrial Radio Access Network (UTRAN) [1], [2].

The UTRAN interconnects the User Equipment (UE) to the Core Network $(\mathrm{CN})$ of the UMTS system. The fundamental components of the UTRAN are the Node B and the Radio Network Controller (RNC) (Figure 1). The Iub is the interface between the Node B and the RNC and the Iur is between two RNCs. The Node B can support one or more radio cells, and controls the radio transmission and reception towards and from the User Equipment (UE) in these radio cells. The $\mathrm{RNC}$ is responsible for controlling the resources associated with a number of Node Bs and for negotiating with the Core Network $(\mathrm{CN})$ various aspects such as those related to QoS. The protocols of the UTRAN are structured in two layers: the radio network layer (RNL) and the transport network layer (TNL). Functions specific to the RNL are independent from the functions related with the specific transport technology used over Iub interface inside TNL. The user plane protocol stack is shown in Figure 1. RLC (Radio Link Control) establishes the RLC connection between UE and RNC. The MAC layer deals with logical channels. It handles the mapping between the logical channels and the transport channels. The output of the MAC layer consists of bursts of Transport Block
(TB) periodically generated every Transmission Time Interval (TTI) of the transport channel. For each transport channel, Frame Protocol (FP) layer assembles the bursts transmitted in one TTI into one FP frame which is transmitted to the TNL. The transport technology connecting the RNC and Node Bs over the Iub interface depends on the choices retained by the operators; Release 99 of the 3GPP standards specifies a transport scheme based on AAL2/ATM technology. Release $5 / 6$ however proposes an all-IP UMTS setting, both in the CN and in the UTRAN. This is further supported by the Mobile Wireless Internet Forum (MWIF) works [2].

As UMTS is evolving toward a future wireless all-IP network, an IP-based UTRAN gives operators flexibility in routing wireless traffic and recreates a wired-like data network environment which drives down the cost of delivering traffic because in today's pre-deployment and deployment phases, operators are more and more aware that their capital expenditure and operating expenses are mostly on the radio access network. IP as the common layer 3 protocol in the UTRAN brings multiple options to an operator in choosing a layer $1 / 2$ backhaul technologies. It is now almost certain that IP will be used in the UTRAN. Therefore, it is necessary to develop transport techniques that can meet QoS requirements in the UTRAN. In this paper, we highlight different architectures and techniques for IP-based transport in the UTRAN.

The remainder of this paper is organized as follows. In Section II, we recall the transport requirements in the UTRAN. In Section III, we briefly describe the multiplexing schemes proposed to fulfill efficient bandwidth utilization. Section IV provides an overview of different QoS capable transport architecture options, such as DiffServ and MPLS. we then discuss the positive aspects and the weakness of the possible transport solutions with multiplexing. In Section $\mathrm{V}$, we investigate the topology of the UTRAN. Section VI briefly concludes the paper.

\section{TRANSPORT QOS REQUIREMENTS}

The 3GPP has specified an open QoS architecture including the definition of four QoS traffic classes: conversational, streaming, interactive and background [4]. The main distinguishing factor between these classes is how delay sensitive the traffic is. Traffic of class conversational is very delay sensitive, such as voice, while Background class is the most 

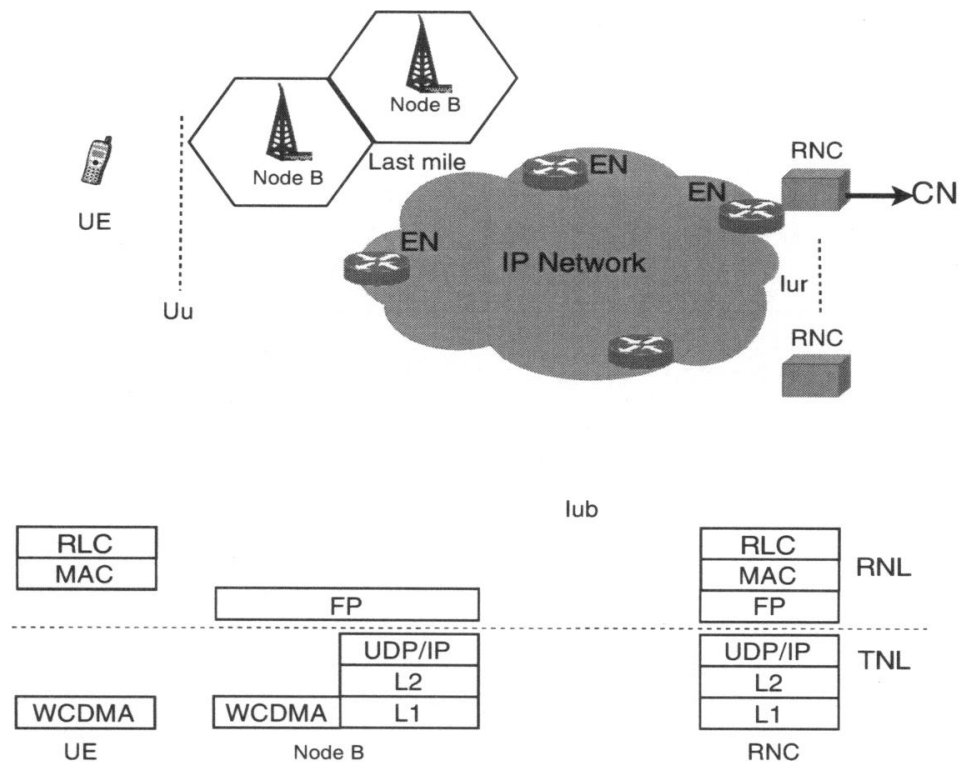

lub

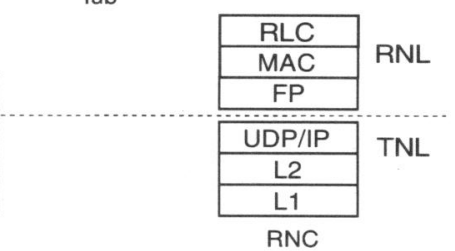

Fig. 1. UTRAN Network and protocol stack

delay insensitive traffic class e.g. E-mail. Conversational and Streaming classes are mainly intended to be used to carry realtime traffic. Interactive and Background classes are mainly meant to be used by non-real time traffic.

To efficiently accommodate the different traffic classes, the transport in the UTRAN should take into account several factors, including the QoS constraints and network topology. Indeed, the advanced radio control functions require that the transport of various types of user traffic, be it real-time or non real-time, over the UTRAN satisfy stringent delay bounds [3]. The hierarchical architecture of the UTRAN makes use of a centralized radio resource management server, which is embedded in the RNC. Due to the fact that the RLC/MAC layers are on RNC side, even the non real-time traffic becomes time constraint traffic over Iub interface in UTRAN, but with more relaxed delay requirements than the real-time traffic.

The UTRAN QoS includes the Radio Access Bearer service (RAB) which provides confidential transport of signaling and user traffic between $\mathrm{UE}$ and the $\mathrm{CN}$ [4]. Between the Node B and the RNC, the RAB service uses the IP-based transport which must satisfy the above-mentioned QoS requirements of user plane traffic. The latter, arrived at the TNL in form of FP frames, shall be put into IP packets. The RNC-Node B connection ranges from a simple E1/T1 link to a MAN networks as well as the existant Internet or any combination of these. Typically the UTRAN is as shown in Figure 1. The last mile to the Node B is typically low speed and may be connected to an egde node (EN) of the UTRAN backbone. Hence, an efficient utilization of the bandwidth is required. In addition to the delay constraints and the efficiency of the network utilization, the transport architecture should present low complexity and moderate cost of the transmission network.

\section{EFFICIENT BANDWIDTH UTILIZATION}

Bandwidth must be used efficiently, especially on the last mile. As UDP/IP layers introduce a significant overhead, multiplexing and header compression reduce the impact of the IP and UDP headers. Several multiplexing schemes have been proposed to reduce the protocol overhead by multiplexing a number of small frames or packets into larger packets [1], [8]. When header compression is applied, the overhead is further significantly reduced. We can classify these multiplexing schemes into two groups: layer 3 versus layer 2 mulyiplexing. The most promising are :

- Composite IP (CIP) is a layer 3 multiplexing scheme proposed by Alcatel in [2]. The FP frames are assembled to fit the CIP packet payload and form a CIP container. A segmentation/re-assembly mechanism allows to split large FP frames into smaller segments. Several CIP containers are multiplexed into one IP packet. The proposed protocol stack is shown in figure $2 \mathrm{a}$.

- Lightweight IP Encapsulation (LIPE) scheme (figure 2b) is also a layer 3 multiplexing scheme proposed in [5] to multiplex low bit rate audio (or multimedia) packets into a single UDP/IP session. It is used and simulated in [2] by Lucent Technologies to multiplex FP frames into IP packets.

- AAL2/IP [6] is again a layer 3 multiplexing scheme (figure 2c) where multiplexing of FP frames into IP packet is carried out above the IP layer by AAL2 layer.

- PPP Multiplexing (PPPmux) [7] is a layer 2 multiplexing scheme (figure 2d) that uses Point-to-Point Protocol. One FP frame is encapsulated into one IP packet with possible UDP/IP header compression (cUDP/IP). The key idea is to concatenate multiple PPP encapsulated frames containing IP packets into a single PPP multiplexed frame. 


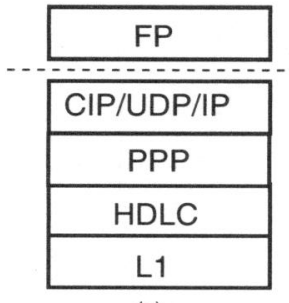

(a)

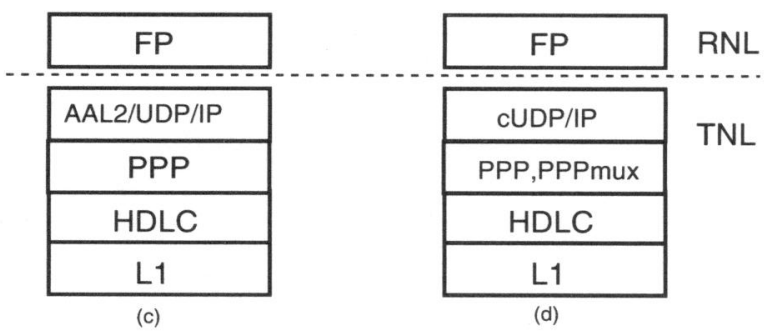

Fig. 2. Protocol stack
RNL

TNL

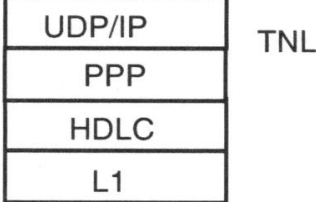

(b)

(d) yields better performance, especially when implemented in an end-to-end fashion between the Node B and the RNC. Endto-end multiplexing is transparent to intermediate transport nodes, i.e. the UTRAN backbone nodes. It provides several advantages such as simplicity, flexibility and security because multiplexing is located in the operator's infrastructure. In the Uplink FP frames are encapsulated into IP packets which are marked in the DS field according to its QoS UMTS classes. In the Downlink, FP frames with the same destination are encapsulated into the same IP packet.

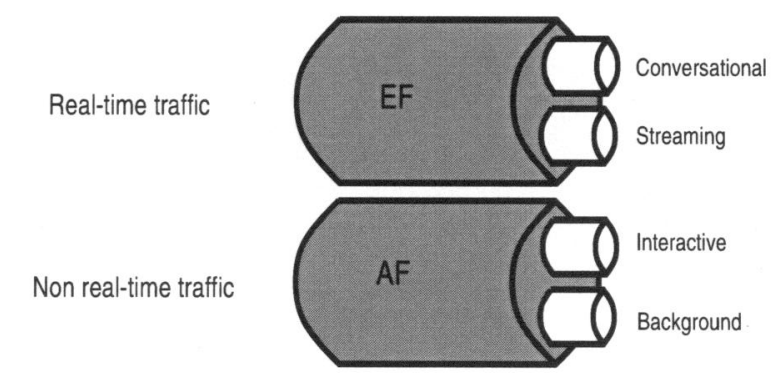

Fig. 3. UTRAN QoS classes

The UTRAN backbone has to support the required QoS and delay bounds. Various QoS models and solutions exist for IP networks to implement an efficient QoS architecture taking into account the UTRAN traffic requirements. Among them, Differentiated Services (DiffServ) and Multi-Protocol label switching (MPLS) are the most promising proposals. At this stage, we evaluate the multiplexing schemes stated earlier in the context of DiffServ and MPLS.

\section{A. DiffServ}

DiffServ is a hop by hop QoS architecture for IP networks. It achieves scalability by aggregating traffic at the edge node of the DiffServ domain and assigning it to a finite number of classes or Per Hop Behaviors (PHB). Core routers implement several prioritisation techniques that reflect those different PHBs. A DiffServ Code Point (DSCP) identifies a PHB and it is set in each packet header. In addition to the Best Effort, two PHBs or classes are defined: The Expedited Forwarding (EF) and The Assured Forwarding (AF). EF is intended to support services with tightly bounded loss, delay and jitter. AF offers different levels of forwarding assurances for packets belonging to an aggregated flow. Packets are marked with one of three drop precedence, such that those with the lowest drop precedence are dropped with higher probability than those marked with the highest drop precedence. DiffServ is already adopted in the UMTS backbone. The UMTS QoS classes are mapped to DiffServ PHBs. Conversational class is mapped to the EF, streaming is mapped to $\mathrm{EF}$ or $\mathrm{AF}$, interactive and background classes are mapped into AF PHBs.

When UTRAN is a DiffServ domain, the Node B is connected to an egde node and the RNC is an edge node or connected to an edge node of the DiffServ Domain. Realtime traffic is transported in the EF class and non real-time is transported in AF class (Figure 3). As DiffServ is used at layer 3, in other words all QoS information is contained in the DSCP, the layer 3 multiplexing, notably CIP and LIPE,
There are other propositions to achieve efficient link utilization such as the multiplexing on the last mile only. This scheme requires additional functionalities at the EN of Node B side to fulfill multiplexing in the downlink and de-multiplexing in the uplink. In addition to the complexity in the transport network, this solution is not flexible if the EN is not a part of the operator network because in public networks this requires tackling issues of security. The same limitations exists if two multiplexing sessions are envisaged, one between Node B and the EN and another one between the EN and RNC.

When layer 2 PPP multiplexing is used, the DiffServ advantage is canceled because IP packets become transparent during the transport, except if the PPP trame is re-encapsulated into an IP packet. This re-encapsulation degrades the multiplexing benefits as it reduces the link utilization efficiency due to the overhead.

\section{B. MPLS}

Multi-Protocol Label Switching (MPLS) integrates network layer routing with label switching forwarding paradigm. It has the ability to build virtual paths called label switched path (LSP) in IP networks [10].

MPLS forwards IP packets based on a label. At the ingress node of the MPLS domain, a label is added to each IP packet containing information that alerts the next hop MPLS node to forward the packet in a pre-defined LSP. The group of packets that is forwarded in the same manner over the same LSP is called a Forwarding Equivalence Class (FEC). The egress node of the MPLS domain removes the MPLS label from each IP packet, and subsequently the IP packets are forwarded by conventional IP forwarding.

In the MPLS-based UTRAN, if multiplexing is done at layer 3 a label is added to each IP packet at the ingress node of the MPLS network. This packet is then forwarded along a pre-defined LSP by performing label swapping instead 
of IP header forwarding. One possibility is that a separate LSPs would be defined between each pair of edge nodes to transport real-time and non real-time traffic. MPLS may support DiffServ by mapping DiffServ aggregates and classes into corresponding LSPs with appropriate QoS guarantees. The DSCP field can be used as a label. In this case, multiplexing is more efficient between the Node B and the RNC rather than on the last mile only for the same reasons steadied in the DiffServ section.

As of multiplexing at layer 2, PPPmux can be used on the last mile only to achieve multiplexing and demultiplexing. In the uplink, the IP packet is decapsulated at the ingress node and a corresponding MPLS label is added. In the downlink, packets having the same destination are encapsulated into a PPP frame at the egde node. When header compression is applied to the IP packet, the IP compressed header can be used as a label. End-to-end layer 2 multiplexing between the RNC and the Node B is proposed in [11] with an optimized protocol stack (Figure 4) using PPPmux. FP frames are encapsulated into IP packets with UDP/IP header compression. The multiplexing layer (UDP/IP/PPPmux) is transported transparently through the intermediate transport nodes thanks to Layer 2 Tunneling Protocol (L2TP). It is only treated in the RNC and the Node B. This requires a re-encapsulation into IP packets so that the tunneling layer (L2TP/UDP/IP) is the layer seen by the UTRAN backbone nodes. This architecture increases the complexity in the transport network and the overhead traffic with reduces efficiency.

\begin{tabular}{|c|c|c|}
\hline FP & & FP \\
\hline cUDP/IP & \multirow{3}{*}{$\begin{array}{c}\text { End-to-end multilplexing } \\
\text { Tunneling }\end{array}$} & cUDP/IP \\
\hline PPPmux & & PPPmux \\
\hline L2TP & & L2TP \\
\hline UDP/IP & & UDP/IP \\
\hline L2 & & L2 \\
\hline $\mathrm{L} 1$ & & L1 \\
\hline
\end{tabular}

Fig. 4. End-to-end layer 2 multiplexing with tunneling

\section{NETWORK TOPOLOGY}

In addition to the schemes analyzed above, the topology of the radio access network might affect the efficiency of the transport solutions. Several topologies can be found in the access network for interconnecting Node Bs and RNC. Figure 5 shows some of the most configurations normally used in the context of the UMTS. When a Node B is an intermediate node in the topology, it will be integrated with an IP router while the end Node B will be equipped with an IP host.

The layer 3 multiplexing can be applied in each Node B. The Node B router forwards the IP packets to the proper outgoing interface into the next hop. This option is compatible with DiffServ as well as MPLS regardless the network topology. The transport resources required in the case (a) are higher than those in the case (b); From economical point of view, the topology that allows high degrees of traffic concentration is attractive as is the case of the topology (c). In this case, the Node B router can acts as a concentrator of all end Node Bs traffic and the multiplexing process can be implemented at this stage to further maximize bandwidth utilization.



Fig. 5. Interconnection topologies

\section{CONCLUSION}

This paper investigated the different QoS architectures with multiplexing schemes for transport of user traffic in the IPbased UTRAN. End-to-end layer 3 multiplexing is best; It is compatible with both hop by hop QoS architecture such as DiffServ and end-to-end QoS architecture like MPLS, with a possibility to run DiffServ over MPLS regardless the implemented topology. PPP layer 2 multiplexing is possible over MPLS LSPs or tunnels established between the Node B and the RNC and best when the setting is that of only a E1/T1 link between the Node B and the RNC.

\section{REFERENCES}

[1] 3GPP TS 25.933 V1.7.1 (2002-01), IP Transport in UTRAN Work Task Technical Report.

[2] Mobile Wireless Internet Forum IP in the RAN as a Transport Option in 3rd Generation Mobile Systems, Technical Report MTR-006, Release v2.0.0, Ratified June 18, 2001.

[3] 3GPP TR 25.853 V3.0.0, Technical Specification Group Radio Access Network; Delay Budget within the Access Stratum.

[4] 3GPP TS 23.107, Technical Specification Group Radio Access Network; QoS Concept and Architecture (Release 5).

[5] M. C. Chuah et al., A LightWeight IP Encapsulation (LIPE) Scheme, Internet Draft, draft-chuah-avt-lipe-00.txt, December 2000.

[6] W. Brunnbauer, G. Cichon, Bringing two worlds together: AAL2 over IP for Radio Access Networks, IEEE Globecom 2001.

[7] R. Pazhyannur, I. Ali, and C. Fox. PPP Multiplexing, IETF RFC 3153, August 2001

[8] A. Samhat, T. Chahed, Transport in IP-based UMTS Radio Access Network: Analytical Study and Empirical Validation, In Proc. IEEE ICC, Paris, June 2004.

[9] A. Samhat, T. Chahed, G. Hébuterne, Priority Queuing for IP-based Service Differentiation in the UMTS Radio Access Network, In Proc. IFIP Networking 2004, Athens.

[10] Y. Guo et al., IP transport in 3G Radio Access Networks: an MPLSbased Approach, Wireless Communications and Networking Conference WCNC, 2002

[11] K. Venken et al., Analysis of the Evolution to an IP-Based UMTS Terrestrial Radio Access Network, IEEE Communications Magazine, October 2003 